the study was to describe the epidemiology of HIV/AIDS in the period 2000-2009.

Methods We conducted a descriptive study using secondary data provided by the National HIV/AIDS Program in Ecuador for the period 2000-2009. The analyses were performed in Epi Info 3.5.2.

Results The general population prevalence of HIV was $0.14 \%$ in the period 2000-2009. In this period there were 5791 AIDS cases and 13567 HIV cases. The incidence rate of HIV/AIDS increased from 5.23 per 100000 inhabitants in 2000 to 38.35 per 100000 in 2009 . During this period, the mortality rate was 5.03 per 100000 inhabitants. In 2009, the HIV prevalence among MSM (men who have sex with men) was $19 \%$, among sex workers $3.2 \%$, and among pregnant women $0.18 \%$.

Conclusion The prevalence of HIV in the general population is low. The results also show an increasing incidence rate of HIV/ AIDS in the period 2000-2009. The mortality rate from HIV was low in relation to the morality rate in Central and South America (14.5 per 100000 inhabitants) for the same period. In MSM, the prevalence of HIV was more than $5 \%$ meeting the criteria of concentrated.

\section{SP4-37 METABOLIC SYNDROME PREVALENCE AND POPULATION ATTRIBUTABLE RISK AMONG HIV/AIDS PATIENTS: COMPARISONS BETWEEN NCEP-ATPIII, IDF AND AHA/NHLBI CRITERIA}

doi:10.1136/jech.2011.142976p.31

\begin{abstract}
${ }^{1,2} \mathrm{P}$ R de Alencastro, ${ }^{*}{ }^{3,4} \mathrm{~S}$ C Fuchs, ${ }^{2} \mathrm{R}$ R de Oliveira, ${ }^{3,4} \mathrm{~F} \mathrm{H}$ Holff, ${ }^{2} \mathrm{M}$ L R lkeda, ${ }^{3,5} \mathrm{~A}$ B M Brandão, ${ }^{3,4} \mathrm{~N}$ T Barcellos. ${ }^{1}$ Postgraduate Studies Program in Medical Sciences, School of Medicine, Universidade Federal do Rio Grande do Sul, Porto Alegre-RS, Brazil; ${ }^{2}$ Hospital Sanatório Partenon, Health Secretariat of State of Rio Grande do Sul, Porto Alegre-RS, Brazil; ${ }^{3}$ National Institute for Science and Technology for Health Technology Assessment (IATS/CNPq), Hospital de Clinicas de Porto Alegre, Porto Alegre-RS, Brazil; ${ }^{4}$ Postgraduate Studies Program in Epidemiology, School of Medicine, Universidade Federal do Rio Grande do Sul, Porto Alegre-RS, Brazil; ${ }^{5}$ Faculdade de Medicina, Universidade Federal de Ciências da Saúde de Porto Alegre, Porto Alegre-RS, Brazil
\end{abstract}

Background Metabolic syndrome comprises a set of aggregated risk factors, which increase the risk of cardiovascular disease and type 2 diabetes mellitus, and changes in the abnormality criteria account for differences in prevalence rates and in the population attributable risk

Objectives Verify the prevalence of metabolic syndrome according to the NCEP-ATPIII, IDF and the AHA/NHLBI, and the impact of each component on the diagnostic of metabolic syndrome among HIVinfected adults. We also estimated the HIV-infected population with metabolic syndrome by Brazilian regions.

Methods This cross-sectional study enrolled HIV infected patients from a HIV/AIDS reference Center in southern Brazil. Metabolic syndrome was identified according to the NCEP-ATPIII, IDF and AHA/NHLBI criteria, using standardised questionnaire and blood testing.

Results A sample of 1240, out of 1295, HIV-infected patients was enrolled. Males were on average older, more educated, and had shorter time since the HIV diagnosis. The population attributable risk (PAR) for waist circumference explained $80 \%$ of the prevalence among men and women (AHA/NHLBI criteria). Triglycerides had the highest impact on prevalence of metabolic syndrome according to all criteria, independently of age, skin colour and HAART use, among men. The southern and south regions of Brazil had the highest population of subjects HIV-infected with metabolic syndrome.

Conclusions In this large sample of HIV infected patients, the overall prevalence of metabolic syndrome, under either classification, was noticeable and the AHA/NHLBI definition accounted for the highest prevalence. The largest population HIV-infected with metabolic syndrome is concentrated in the southern and south of Brazil.

\section{SP4-38 IMPACT OF TOOTH BRUSHING IN PLAQUE INDEX IN ADOLESCENTS}

doi:10.1136/jech.2011.142976p.32

I Bica, ${ }^{*}$ M Cunha, J Costa, V Rodrigues, P Costa. Superior Health School of Viseu, Polytechnic Institute of Viseu, Viseu, Portugal

Objectives To evaluate the plaque index and oral hygiene habits of adolescents.

Methodology Tranversal and descriptive study in a sample of 189 adolescents aged between 11 and 17 years of age. Data collection was conducted through a questionnaire to adolescents about oral hygiene. There has yet to assess the plaque index simplified by applying the developer board.

Results For the Plaque Index Simplified the vast majority $(98.9 \%)$ of adolescents reveals plaque. Most (69.3\%) of teens brush their teeth twice a day, $54.5 \%$ of those referred to brush your teeth, tongue and gums and $60.8 \%$ state that it takes $2-5$ min to perform brushing. However $36 \%$ never runs brushing at night. The deficient oral hygiene habits (46.6\%) and good oral hygiene habits (46\%) among the adolescents are very similar.

Conclusion The results suggest that there is need to develop with teenagers individualised training and supervision of proper brushing of teeth.

\section{SP4-39 NUTRITIONAL VIGILANCE SYSTEM OF COMMUNITY: A LOT QUALITY ASSURANCE SAMPLING FOR MONITORING COVERAGE OF A NUTRITIONAL KNOWLEDGE AND ATTITUDES CLUES FROM MOTHERS OF CHILDREN 0 TO 23 MONTHS OF AGE IN BOLIVIA}

doi:10.1136/jech.2011.142976p.33

${ }^{1,2} \mathrm{H}$ Castillo, ${ }^{* 1,2} \mathrm{~K}$ I Candia, ${ }^{1,2} \mathrm{~A}$ Sucre, ${ }^{1,2} \mathrm{~A}$ M Aguilar. ${ }^{1}$ Ministry of Health and Sport, La Paz, La Paz, Bolivia; 'ZZero Malnutrition Program, La Paz, La Paz, Bolivia

Background This study reports on the results of a largescale targeted of nutritional promotional interventions in Bolivian municipalities with high Food Insecurity and Vulnerability classification.

Methods The lot quality assurance sampling (LOAS) method was used for the assessment of the geographical municipalities' coverage in Bolivia, as part Zero Malnutrition Program a government politics, between April and December of 2010. The mothers of children 0 and 23 months of age of 134 municipalities were interview using a structured questionnaire. The municipalities had a four and five score of Food Insecurity and Vulnerability classification. The collet data for this study, 134 interview teams were hired. The coordination of teams received $32 \mathrm{~h}$ of training. During data collection (April to November 2010), 22 technical advisors provided supervision and technical support. Part of the mothers was interviewed in language native.

Results The significant general decrease in the coverage proportions of nutritional attitudes indicators in relation to knowledge indicators was observed. The coverage proportions of any breastfeeding and the exclusive breastfeeding attitude decrease in comparison with the knowledge indicators, the same form with the complementary alimentary (Nutribebe ${ }^{\circledR}$ and Chispitas ${ }^{\circledR}$ ).

Conclusion The LOAS incorporate in the Nutritional Vigilance System of Community is seen as a valuable tool for the routine monitoring of the geographical coverage and permit identify quantitative differences between attitudes and knowledges in mothers of children 0 and 23 months of age from which is was possible to reformulate of nutritional promotional interventions in the municipality. 